\title{
Effect of the electric field on the carrier collection efficiency of InAs quantum dots
}

\author{
(C) E.S. Moskalenko*,**, K.F. Karlsson*, V. Donchev ${ }^{* * *}$, P.O. Holtz*, W.V. Schoenfeld ${ }^{* * * *}$, P.M. Petroff**** \\ * Department of Physics and Measurement Technology, Linköping University, \\ S-58183 Linköping, Sweden \\ ** A.F. loffe Physical-Technical Institute, Russian Academy of Sciences, \\ 194021 St. Petersburg, Russia \\ *** Faculty of Physics, Sofia University, \\ 1164 Sofia, Bulgaria \\ **** Materials Department, University of California, Santa Barbara, \\ 93106 California, USA
}

E-mail: evgenii.moskalenko@mail.ioffe.ru

(Received January 17, 2005)

\begin{abstract}
Individual and multi quantum dots of InAs are studied by means of microphotoluminescence in case when, in addition to the principal laser exciting photoluminescence, second infrared laser is used. It is demonstrated that the absorption of the infrared photons effectively creates free holes in the sample, which leads to both change in the charge state of a quantum dot and to the considerable reduction of their photoluminescence signal. The later effect is explained in terms of an effective screening of the internal electric field, facilitating the carrier transport along the plane of a wetting layer, by the surplus holes from the infrared laser. It is shown that the effect of quenching of quantum dot photoluminescence gradually disappears at increased sample temperature $(T)$ and / or dot density. This fact is due to the essentially increased value of quantum dot collection efficiency which could be achieved at elevated sample temperatures for the individual quantum dots or even at low $T$ for the case of multi quantum dots. It is suggested that the observed phenomena can be widely used in practice to effectively manipulate the collection efficiency and the charge state of quantum dot-based optical devices.
\end{abstract}

E.S.M. gratefully acknowledges financial support from the Wenner-Gren Foundations and partial support from the program „Low-Dimensional Quantum Structures“ of the Russian Academy of Sciences. V.D. is thankful to the Swedish Foundation for International Cooperation in Research and Higher Education (STINT) for financial support.

\section{Introduction}

Semiconductor quantum dots (QD's) effectively confine electrons $(e$ 's) and holes $(h$ 's) on the nanometer length scale in all three dimensions and hence may be considered as „artificial atoms“ [1]. Unlike real atoms, QD's can be manipulated in different ways, which opens the possibility to tailor their shape, size and composition [2] in order to achieve the desired properties. Consequently, QD's are potential candidates for various optoelectronic (electronic) applications such as QD lasers [3], QD infrared detectors [4], QD memory devices [5] and single-electron transistors [6].

For a majority of these devices the QD's become populated with carriers, which are primarily created outside the QD's somewhere in the sample (in the barriers or in the wetting layer (WL), on which QD's are normally grown [7]) by means of electrical or optical excitation. Consequently, excited carriers undergo a transport in the $\mathrm{WL} /$ barriers prior to the capture into the QD's. This circumstance highlights the crucial role of the carrier capture processes into the QD for the performance and operation of the QD-based devices.

The carrier capture mechanisms intensively studied in the last decade reveal optical phonon assisted [8,9], Augerlike [10], shake-up [11] processes and carrier relaxation through the band tail states of the WL with a subsequent emission of localized phonons [12]. The lateral carrier transport (in the plane of the WL) could be affected by carrier hopping between QD's [13], by trapping of migrating particles into localized states of the WL [14] or into nonradiative centers [15] in the surrounding media. A more efficient carrier transfer from the WL into the QD's via radiation-induced defects in the WL has been reported [16]. A magnetic field directed perpendicular to the plane of the structure was observed to limit the lateral transport of carriers [17].

It has also been suggested [8] that the carrier drift could be considerably influenced by a long-range attractive potential caused by the strain field surrounding the QD. On the other hand, strain-induced potential barriers in the barrier/QD [18] and in the WL/QD interface [19] were considered to limit the carrier capture into the QD. The important role of an electric field directed in the growth direction of the sample on the carrier capture into and escape out of the QD was demonstrated by the studies of the electric current passing through the QD's [20].

In our previous study [21] we pointed out another mechanism of the carrier transfer from the WL into the QD's, which has not been previously considered. A built-in electric field $(F)$ directed in the plane of a WL to facilitate the lateral carrier transport. However, in these measurements individual QD's were studied only at a fixed sample temperature $(T)$ of $5 \mathrm{~K}$. In the present paper the suggested mechanism on the carrier capture into the QD's is investigated at increased sample temperatures (up to $70 \mathrm{~K}$ ) as well as increased dot density. 
In our experiments we use an additional infrared (IR) laser to influence the field $F$. The excitation energy of the IR laser, $h v_{\mathrm{IR}}=1.240 \mathrm{eV}$, is considerably less than the lowest transition energy of the sample studied and, accordingly, can not simultaneously excite both electrons $(e$ 's) and holes $(h$ 's), but can generate solely either $e$ 's or $h$ 's by excitation of deep level (DL) defects positioned in the band gap of the CaAs barriers [22]. According to our model, these extra carriers, excited by the IR laser, will effectively screen the field $F$ and will consequently slow down the carrier transport in the plane of the WL. Due to this effect, a considerable reduction (up to 10 times) of QD photoluminescence $(\mathrm{PL})$ signal $\left(I_{\mathrm{QD}}\right)$ is experimentally observed when the sample is exposed to dual excitation of an IR laser and a main laser.

To the best of our knowledge, there are very few earlier publications $[23,24]$ devoted to studies of IR laser induced changes in $I_{\mathrm{QD}}$. In contrast to our findings, it was found [23] that the IR laser induces an increase of the PL from the QD's by up to $40 \%$. This phenomenon was explained in terms of an IR laser induced release of carriers, which were trapped into deep defects from the QD's. The considerable changes in the fluctuations of the $I_{\mathrm{QD}}$ during the time interval of the measurement were detected, when the sample was illuminated with an additional near-IR laser irradiation [24]. Carriers trapped at deep localized centers in the vicinity of the QDs were suggested to be responsible for the observed phenomenon [24].

Our present results demonstrate that the strength of the observed quenching effect of $I_{\mathrm{QD}}$ progressively decreases with an increasing temperature as well as dot density. This is explained in terms of an essentially increased QD's collection efficiency $(\alpha)$, i. e. the ability of QD's to collect photoexcited carriers from illuminated area. At these experimental conditions the role of $F$, which facilitates the carrier transport at a lower values of $\alpha$, becomes diminishing.

\section{Sample and experimental setup}

The sample studied was grown by molecular beam epitaxy on the GaAs (100) substrate. It consists of lensshaped InAs QDs developed on a InAs WL from about 1.7 monolayer InAs deposited in Stranski-Krastanov growth mode. The WL and dot layer was sandwiched between two $100 \mathrm{~nm}$ thick GaAs barriers. The sample was grown without rotation of the substrate, resulting in a gradual variation of In flux across the wafer and consequently a gradient in the QDs density. The QDs were studied by means of a diffraction-limited micro-PL $(\mu \mathrm{PL})$ setup (detailed description of the setup and the sample growth procedure is given in [25]). The $\mu \mathrm{PL}$ technique empoyed in the present experiments allowed us to excite and study single QD (SQD).

To excite the sample we used two Ti-Sp lasers, which beams were focused on the same position of the sample surface down to a spot diameter of $2 \mu \mathrm{m}$. The main laser $\left(L_{0}\right)$ was used to excite the PL of the WL and the QDs. The exitation energy $\left(h v_{\mathrm{ex}}\right)$ was tuned in the range from 1.410 to $1.480 \mathrm{eV}$ with a maximum excitation power $\left(P_{0}\right)$ of $20 \mu \mathrm{W}$. The other laser, $L_{\mathrm{IR}}$, operating at a fixed excitation energy, $h \nu_{\mathrm{IR}}=1.240 \mathrm{eV}$ has its maximum output power $\left(P_{\mathrm{IR}}\right)$ of $100 \mu \mathrm{W}$. It is important to note that the $h v_{\mathrm{IR}}$ is well below the value of the QDs related emission and accordingly no signal neither from the WL nor from the QDs was detected with excitation solely with $L_{\mathrm{IR}}$. The sample was positioned inside a continuous-flow cryostat operating in a temperature $(T)$ range of 5-70 K.

To find the particular QD to study, a laser beam was scanned across the sample surface. Once the desired QD was found, special marks (grids) were burnt into the sample surface around the QD with a high power laser beam. The average distance between the adjacent QD's in the low dot density area of the sample was around $10 \mu \mathrm{m}$. To control the exact position of the laser spot on the sample surface, the image of the interesting region was projected by a video camera, which made it easy to find the desired QD marked by the grid. In addition, this arrangement allowed us to effectively correct the laser position on the sample, if the sample was moved due to the thermal drift. It should be noted that the method to locate the exact QD position by using the described grids is favourable in several respects compared to alternative methods, e.g. employing a metal mask with small holes deposited on top of the sample which may produce an electric field in the near-surface region and, consequently, may influence the carrier transport in the plane of the WL. In addition, the metal mask may act as a stressor, which could spoil the quality of the QD's.

Eight SQDs located at different spatial positions of the sample, all with an analogous behavior with respect to the $L_{\mathrm{IR}}$, were examined in this study. In this report we present data measured on low density structure with one SQD within the laser spot together with a high dot density area with a varying number of QDs within the area of the laser spot.

\section{Experimental results and discussion}

The low-temperature $\mu \mathrm{PL}$ spectrum of a sample spot with only one QD (SQD) within the laser illumination obtained under excitation with a single laser $L_{0}$ at an excitation energy $h v_{\mathrm{ex}}=1.471 \mathrm{eV}$ is shown in Fig. 1 (dotted lines). The $\mu \mathrm{PL}$ spectrum of the sample is dominated by the WL emission at an energy around $1.445 \mathrm{eV}$. A weak emission line, peaking around $1.340 \mathrm{eV}$ is attributed to the PL from a $\mathrm{SQD}$. The spectrally integrated PL signal from a SQD $\left(I_{\mathrm{QD}}\right)$ is approximately $1 \%$ with respect to the corresponding $\mathrm{PL}$ intensity of the $\mathrm{WL}\left(I_{\mathrm{WL}}\right)$. This ratio is much higher than the corresponding volume ratio $\left(\approx 10^{-3}\right)$ of the SQD vs the excited WL volume. This fact is a direct evidence that the PL signal from a SQD is not entirely determined by the number of carriers excited in the SQD as a result of 


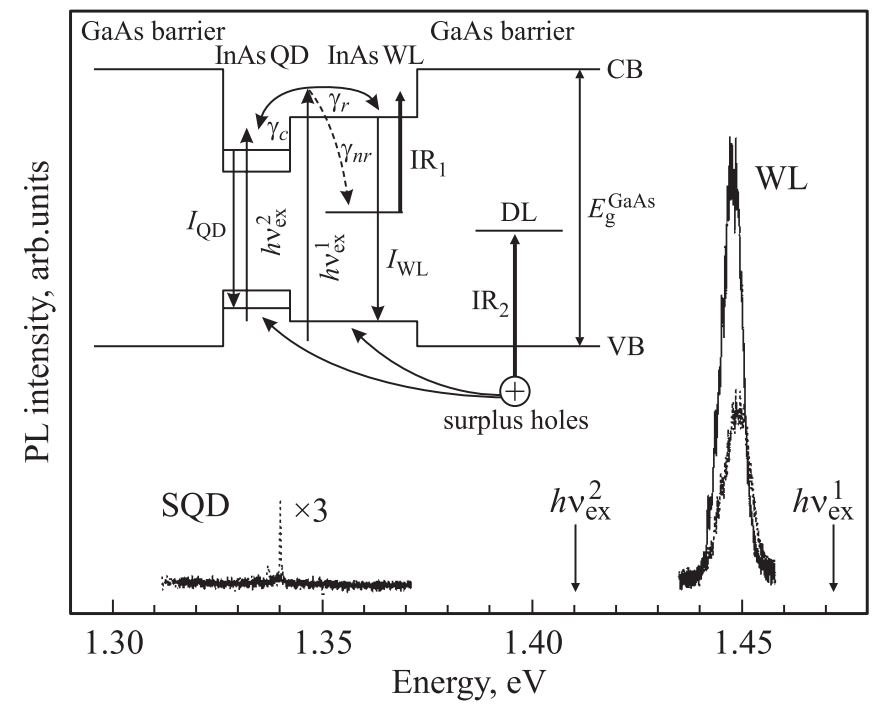

Figure 1. $\mu \mathrm{PL}$ spectra of a SQD and the WL measured at $T=5 \mathrm{~K}$ and $h v_{\mathrm{ex}}=1.471 \mathrm{eV}$ with single (dotted lines) and dual (solid lines) laser excitation, respectively, at an excitation power of $P_{0}=200 \mathrm{nW}$ and $P_{\mathrm{IR}}=100 \mu \mathrm{W}$. The vertical solid arrows show the excitation energies of $L_{0}$ used in the experiment. The inset shows the principle transitions involved in the energy scheme of the sample together with the positions of the GaAs conduction band (CB), valence band (VB) and deep level (DL). The vertical and curved arrows are explained in the text.

absorption of photons in the dot volume, but rather to a significant extent by carriers excited in the WL. In other words, the WL serves as a reservoir which supplies the QD with carriers. Indeed, carriers excited in the WL $\left(h v_{\mathrm{ex}}^{1}\right.$ in the inset in Fig. 1) can undergo alternative trapping processes: a) a relaxation down to the localized WL states, followed by a radiative recombination, b) a trapping at centers of nonradiative recombination (CNRs) or c) capture into a SQD (processes shown by the arrows $\gamma_{r}, \gamma_{n r}$ nad $\gamma_{c}$, respectively, in the inset in Fig. 1). The interplay between these processes determines the values of $I_{\mathrm{QD}}$ and $I_{\mathrm{WL}}$ measured in the experiment.

We have solved a simple set of rate equations in steady-state conditions (not shown here) which resulted in $I_{\mathrm{QD}} / I_{\mathrm{WL}}=\gamma_{c} / \gamma_{r}=\alpha$ at any value of $\gamma_{n r}$, where the parameter $\alpha$ is denoted the collection efficiency of the SQD. The physical meaning of the introduced parameter $\alpha$ could be explained by the following example. If the capture probability $\gamma_{c}$ exceeds the value of $\gamma_{r}$, the PL of the SQD would dominate the PL spectrum of the sample at the expense of the WL PL, i. e. the dot would efficiently collect a majority of carriers created by the laser absorption within the laser spot.

A spectacular effect was observed when, in addition to the excitation of $L_{0}$ at $h v_{\mathrm{ex}}=1.471 \mathrm{eV}$, the sample was illuminated with an infra-red laser, $L_{\mathrm{IR}}$. Indeed, the $\mu \mathrm{PL}$ spectrum of the sample (solid lines in Fig. 1) undergoes a dramatic change in this case: While $I_{\mathrm{WL}}$ increases by a factor of 2.5, the SQD emission almost completely vanishes.
The increase of the $I_{\mathrm{WL}}$ could be explained in terms of a release of carriers from the centers of non-radiative recombination as a result of the infrared absorption by $L_{\mathrm{IR}}$ (arrow IR1 in the inset in Fig. 1). Such an enhancement of the radiative efficiency, induced by an additional below band gap excitation, has been reported earlier (see e.g. [26]). If this was the only operating mechanism caused by $L_{\mathrm{IR}}$, an increase of $I_{\mathrm{QD}}$ would also be expected, since the WL serves as a reservoir of carriers shich could be trapped into the SQD, as explained above. Consequently, a different mechanism of the $L_{\mathrm{IR}}$ influence on the sample has to be considered in order to explain the observed decrease of $I_{\mathrm{QD}}$.

To understand the origin of infrared laser effect on the PL intensity we studied the temperature $(T)$ dependence of the observed effect. Fig. 2 a shows a number of pairs of SQD $\mu \mathrm{PL}$ spectra recorded with single laser $L_{0}$ excitation (dotted lines) and dual laser excitaion (solid lines) at different $T$. Two emission lines marked as $X$ and $X^{-}$have earlier been interpreted [25] as excitonic lines with different charge configurations. The neutral and single negatively charged excitons, i.e. $1 e 1 h$ and $2 e 1 h$ charge states, respectively, could be detected. The progressive redistribution of the $\mu \mathrm{PL}$ spectra in favour of the $X^{-}$line, detected with single laser $L_{0}$ excitation with increasing $T$ (dotted lines in Fig. 2,a) was explained [27] in terms of a temperatureinduced increased electron diffusivity in the WL plane. This leads to a faster diffusion and, hence, capture of $e$ 's into the dot with respect to the capture of $h$ 's. The $\mu \mathrm{PL}$ spectra
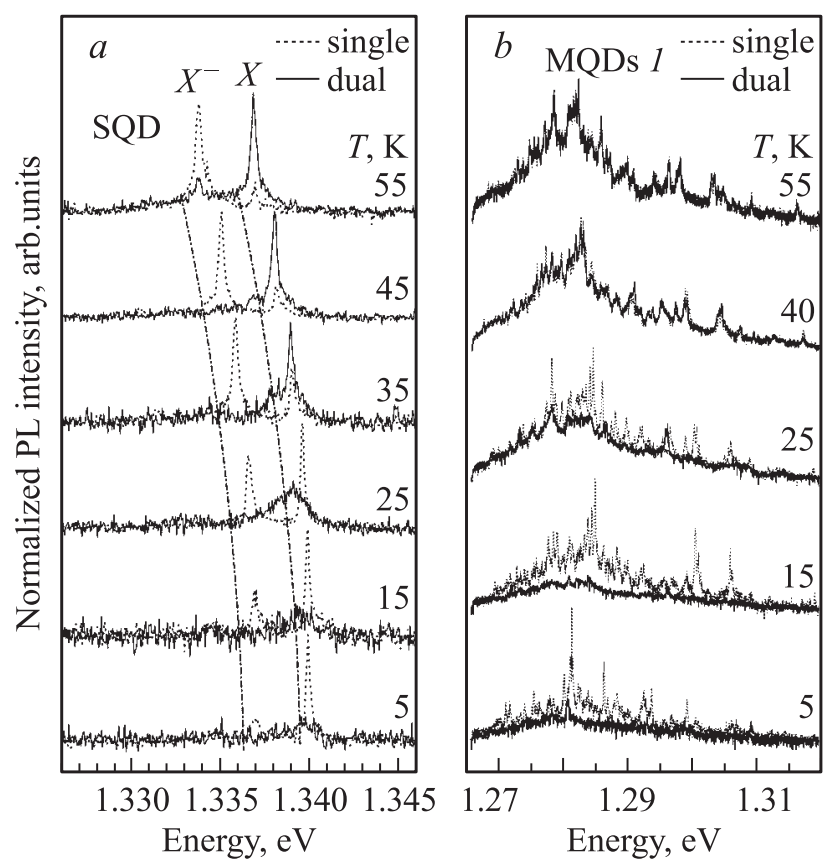

Figure 2. $\mu \mathrm{PL}$ spectra of SQD $(a)$ and MQDs $1(b)$ measured at $h v_{\mathrm{ex}}=1.471 \mathrm{eV}, h v_{\mathrm{IR}}=1.240 \mathrm{eV}$, for a number of temperatures as indicated in the figure and at excitation with a single (dotted lines) and double (solid lines) laser, respectively at an excitation power of $P_{0}=200 \mathrm{nW}$ and $P_{\mathrm{IR}}=100 \mu \mathrm{W}$. The curved dash-dotted lines in $(a)$ are guides for the eye. 

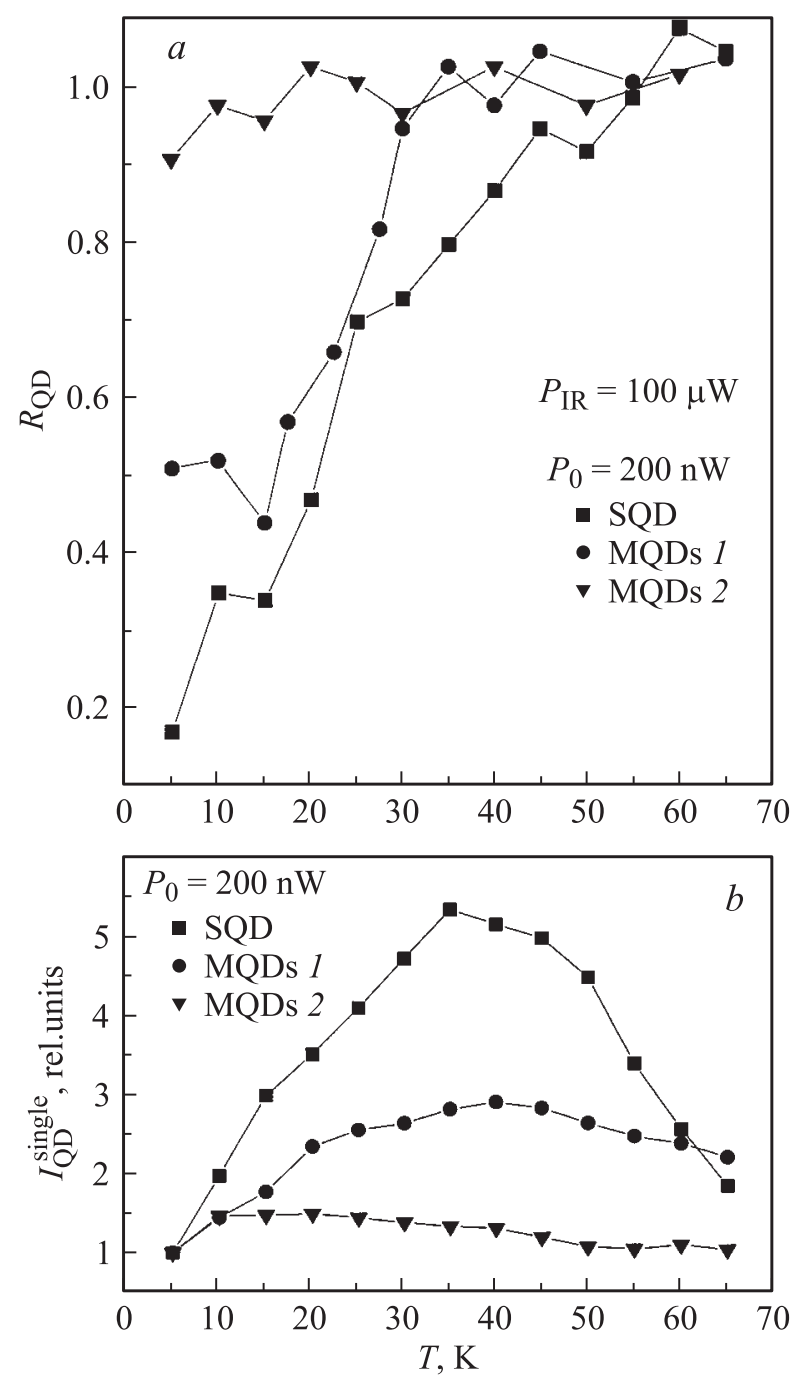

Figure 3. $R_{\mathrm{QD}}(a)$ for $h v_{\mathrm{IR}}=1.240 \mathrm{eV}$ and $P_{\mathrm{IR}}=100 \mu \mathrm{W}$ and normalized values of $I_{\mathrm{QD}}^{\text {single }}(b)$ for SQD, MQDs 1 and MQDs 2 measured for a number of $T$ 's at $h v_{\mathrm{ex}}=1.471 \mathrm{eV}$ and $P_{0}=200 \mathrm{nW}$ shown by solid squares, circles and triangles, respectively.

measured with dual laser excitation (solid lines in Fig. 2, $a$ ) reveal both a redistribution of the emission lines in favour of the $X$ line and a progressively vanishing quenching effect with increasing $T$.

The redistribution effect in favour of $X$ at elevated temperatures is clearly illustrated in Fig. 2,a. This fact clearly demonstrates that $L_{\mathrm{IR}}$ supplies the sample with extra $h$ 's which can be captured by the QD, and accordingly effectively "neutralizing" its charge configuration. The generation of extra $h$ 's is assumed to take place in the GaAs barriers as a result of an IR laser induced electron excitation from the GaAs valence band into DLs always present in the GaAs bandgap [22] (arrow $\mathrm{IR}_{2}$ in the inset in Fig. 1). It is interesting to note that the PL spectrum measured under dual-laser excitation at $T=25 \mathrm{~K}$ is very broad and a peak appears between the $X$ and $X^{-}$lines (Fig. 2,a).
This experimental finding is unclear and further studies are needed to reveal its nature. However, some insight which can elucidate the situation can be achieved from spectral diffusion - a phenomenon previously observed in single self-assembled quantum dots $[28,29]$. In particular, it was found that the electric field induced by the charges, which are located in the close vicinity of a QD, initiated an energy shift of the QD spectral lines, which were quasi-periodic in time $[28,29]$. Consequently, the widths of the spectral lines registered in the time-integrated regime (as in the present manuscript) is expected to broaden in case of the existence of quasi-periodic (in time) local electic fields around the SQD under study [29]. That is why the experimentally observed broadening of the QD PL line and its energy shift detected in the temperature range around $T=25 \mathrm{~K}$ (Fig. 2,a) could be regarded as an evidence for the presence of a local electric field arond the QD.

The effect of change of $I_{\mathrm{QD}}$ induced by the IR laser could quantitatively be described in terms of a „quenching rate", $R_{\mathrm{QD}}$, which is defined as a ratio of $I_{\mathrm{QD}}$ measured with dual laser excitation $\left(I_{\mathrm{QD}}^{\text {dual }}\right)$ to be compared with the corresponding single laser excitation $\mathrm{PL}$ intensity $\left(I_{\mathrm{QD}}^{\text {signle }}\right)$. $R_{\mathrm{QD}}$ values measured for a SQD are shown by solid squares in Fig. 3,a. It is seen that $R_{\mathrm{QD}}$ being as low as 0.1 at $T=5 \mathrm{~K}$, progressively increases to reach the value of 1 at $T \approx 60 \mathrm{~K}$, i. e. the quenching effect gradually disappears with increasing $T$.

A qualitatively similar behaviour could be recorded for another sample spot with a higher dot density (MQDs 1). The $\mu \mathrm{PL}$ spectra in this case consist of a number of sharp lines superimposed on a broad PL band in the spectral range of $1.270-1.310 \mathrm{eV}$ (Fig. 2, b). It is clearly seen that the IR laser also induces a quenching of the PL signal at low temperatures. The quenching rate is found to increase from $R_{\mathrm{QD}} \approx 0.5$ at $T=5 \mathrm{~K}$ with increasing temperature to reach $R_{\mathrm{QD}} \approx 1$ already at $T \approx 30 \mathrm{~K}$ (solid circles in Fig. 3,a). This $T$ is essentially lower than the temperature needed to completely cancel the quenching for the SQD $(60 \mathrm{~K})$ (Fig. 3, a).

In sharp contrast to these observations, similar quenching effects were practically not registered at a sample position with even higer dot density (MQDs 2 ). In fact, $R_{\mathrm{QD}} \approx 0.9$ already at $T=5 \mathrm{~K}$ (solid triangles in Fig. 3, $a$ ) and stays at a value around 1 for $T>5 \mathrm{~K}$. In the following we will present a qualitative model which explains the quenching phenomenon as well as its disappearance at increased temperature and dot density.

To explain the quenching effect we note first that at an excitation energy of $h v_{\mathrm{ex}}=1.410 \mathrm{eV}$ of the principal laser $L_{0}$ (the vertical solid arrow $h v_{\mathrm{ex}}^{2}$ in the inset in Fig. 1) IR laser had no effect on $I_{\mathrm{QD}}$ (results not shown here). It means that $L_{\mathrm{IR}}$ can not influence the number of $e-h$ paris which already have been captured into the QD (at these experimental conditions $e$ 's and $h$ 's are not subjected to transport along the plane of the WL prior to capture into the QD). Consequently, the reason for the observed 
decrease of $I_{\mathrm{QD}}$, registered for an excitation energy of $h v_{\mathrm{ex}}=1.471 \mathrm{eV}$, is the influence of the $L_{\mathrm{IR}}$ on the transport properties of the carriers in the WL plane. Secondly, $I_{\mathrm{QD}}$ is proportional to the collection efficieny $\alpha$ as was explained above. Accordingly the observed decrease of $I_{\mathrm{QD}}$ means a considerabel reduction of $\alpha$ measured under dual laser excitation $\left(\alpha^{\text {dual }}\right)$ with respect to the corresponding single laser excitation conditions $\left(\alpha^{\text {single }}\right)$. Thirdly, as was stated above, $L_{\mathrm{IR}}$ supplies the sample with excess $h$ 's. Consequently, the model suggested should explain how the apperance of the surplus $h$ 's can affect the carrier transport in the WL plane.

We propose the following model to explain the influence of the extra charge on the collection efficiency of the photocreated carriers from the WL into the QD. The existence of an electric field $F$ in the plane of the WL is assumed. Photo-excited $e$ 's and $h$ 's, generated at arbitrary spots within the laser illumination, move along the plane of the WL for some time $(\tau)$ decreasing their kinetic energy until they bind together and recombine as excitons, contributing to $I_{\mathrm{WL}}$. The QD can capture carriers/excitons only for the case, when the capture time from the WL into the QD is less than $\tau$. Accordingly, a rather high velocity is needed to have a non-vanishing probability for carrier transport and capture into QD. The carriers are assumed to achieve a rather high velocity with respect to the thermal velosity when their transport is influenced by the field $F$.

The origin of the built-in field is at present not known and further studies are needed to reveal its nature. The origin is believed to be due to donors and acceptors which are positioned in the vicinity of the QD $[15,16]$. An $e$ from the donor atom can be captured by an acceptor, giving rise to a built-in field $F$ with a component along the WL plane as a result of the charge separation. When the surplus $h$ 's, photoexcited by the $L_{\mathrm{IR}}$, appear in the WL, the carriers will move along the direction of the built-in field. If these extra $h$ 's are localized at the interface potential fluctuations, they could stay there for a rather long time (until an $e$ to recombine with is approaching), providing an effective screening of the field $F$. (A more detailed discussion of the screening mechanism is given in [21]). Consequently, the transport of $e$ 's and $h$ 's, excited in the WL by $L_{0}$ and $L_{\mathrm{IR}}$ will be determined by the thermal velocity which is assumed to be essentially lower than the drift velocity at the unscreened field conditions, and accordingly a decrease of $I_{\mathrm{QD}}\left(R_{\mathrm{QD}}<1\right)$ is expected.

It is important to note that the absolute value of $R_{\mathrm{QD}}$ measured at given experimental conditions (with respect to $P_{0}, P_{\mathrm{IR}}$ and $T$ ) for other individual SQDs was found to exhibit variations depending on the particular SQD under study. This is reasonable since the concentration and space distribution of impurity atoms in the close vicinity of a given SQD, which determines the value of $F$, should have variations depending on their exact location in the sample.
The experimentally measured $R_{\mathrm{QD}}$ can be expressed in terms of collection efficiencies in the following way:

$$
R_{\mathrm{QD}}=\frac{\alpha^{\text {dual }}}{\alpha^{\text {single }}} \cdot R_{\mathrm{WL}}
$$

where

$$
R_{\mathrm{WL}}=\frac{I_{\mathrm{WL}}^{\text {dual }}}{I_{\mathrm{WL}}^{\text {single }}}
$$

$I_{\mathrm{WL}}^{\text {signle }}\left(I_{\mathrm{WL}}^{\text {dual }}\right)$ is the WL PL intensity with single (dual) laser excitation. To explain the observed increase of $R_{\mathrm{QD}}$ with increasing $T$ (Fig. 3,a) we need, according to Eq. 1a, information on temperature evolution of both $R_{\mathrm{WL}}$ and $\alpha^{\text {dual }} / \alpha^{\text {single }}$.

Fig. 4, $a$ shows $R_{\mathrm{WL}}$ for a SQD measured as a function of temperature. It is seen that its value is progressively reduced down to $R_{\mathrm{WL}} \approx 1$ at elevated $T$ 's. Consequently, according to Eq. 1a, the only reason for the observed increase of $R_{\mathrm{QD}}$ (Fig. 3, $a$ ) is the increase of $\alpha^{\text {dual }} / \alpha^{\text {signle }}$ as $T$ becomes higher. Obviously there could be two basically different reasons for the increase of $\alpha^{\text {dual }} / \alpha^{\text {signle }}: \alpha^{\text {single }}$ reduces down to $\alpha^{\text {dual }}$ as $T$ increases or, on the contrary, $\alpha^{\text {dual }}$ could increase significantly to reach the value of $\alpha^{\text {single }}$ at elevated temperatures. The first possibility would imply a temperature-induced screening of the built-in electric field, which facilitates carrier capture into the QD. The second possibility would correspond to the experimental situation that the carrier capture into the QD at high $T$ 's becomes so effective that the role of the field $F$ for the carrier transport progressively diminishes with temperature.

To distinguish between these two possibilities we have studied the temperature evolution of $I_{\mathrm{QD}}^{\text {signle }}$ and $I_{\mathrm{WL}}^{\text {single }}$ (Fig. 4, $b$ ). It is seen that with increasing $T I_{\mathrm{WL}}^{\text {single }}$ is progressively reduced (by more than two orders of magnitude for the total $T$ range studied), while $I_{\mathrm{QD}}^{\text {single }}$ reveals an essential increase ( $>5$ times) when $T$ changes from 5 up to $40 \mathrm{~K}$ to dominate the $\mu \mathrm{PL}$ spectrum of a sample for $T>40 \mathrm{~K}$. The considerable decrease of $I_{\mathrm{WL}}^{\text {single }}$ (Fig. 4, $b$ ) could be explained in the following way. At low $T$ 's photoexcited carries are captured into localized states of the WL (processes shown by arrow $\gamma_{r}$ in the inset in Fig. 1) and stay there until they recombine radiatively contributing to the PL signal of the WL (arrow $I_{\mathrm{WL}}$ in the inset in Fig. 1). The existence of such localized states in the WL is evidenced by the observation of a number of sharp peaks (separated from each other by $<1 \mathrm{meV}$ ) superimposed on the low-energy tail of the WL emission band (Fig. 1). As $T$ increases, carriers become delocalized and their thermal velocity increases. These two effects result in a more efficient transport of carriers along the plane of the WL. Consequently, the probability for carrier to approach a QD (proportional to $\gamma_{c}$ ) or alternatively a CNR (proportional to $\gamma_{\mathrm{nr}}$ ) is considerably increased at elevated $T$ 's. This eventually results in both processes. The increased probability for capture at a CNR will contribute to the quenching of the WL PL, while increased probability to become trapped in a QD will contribute to the enhancement 
of the SQD PL signal (Fig. 4,b). The crucial role of the carrier transport prior to the trapping into the CNR has been described elsewhere [30].

The observed temperature dependence of the $R_{\mathrm{WL}}$ (Figl. 4, a) can be qualitatively explained in terms of an essentially increased value of $\gamma_{\mathrm{nr}}$ at elevated $T$ 's. Indeed, at low $T$ 's, i.e. low values of $\gamma_{\mathrm{nr}}$, carriers which are released from the CNRs (as a result of influence of $L_{\mathrm{IR}}$ ) were captured into localized states of the WL prior to capture back to the CNRs. This resulted in the increase of
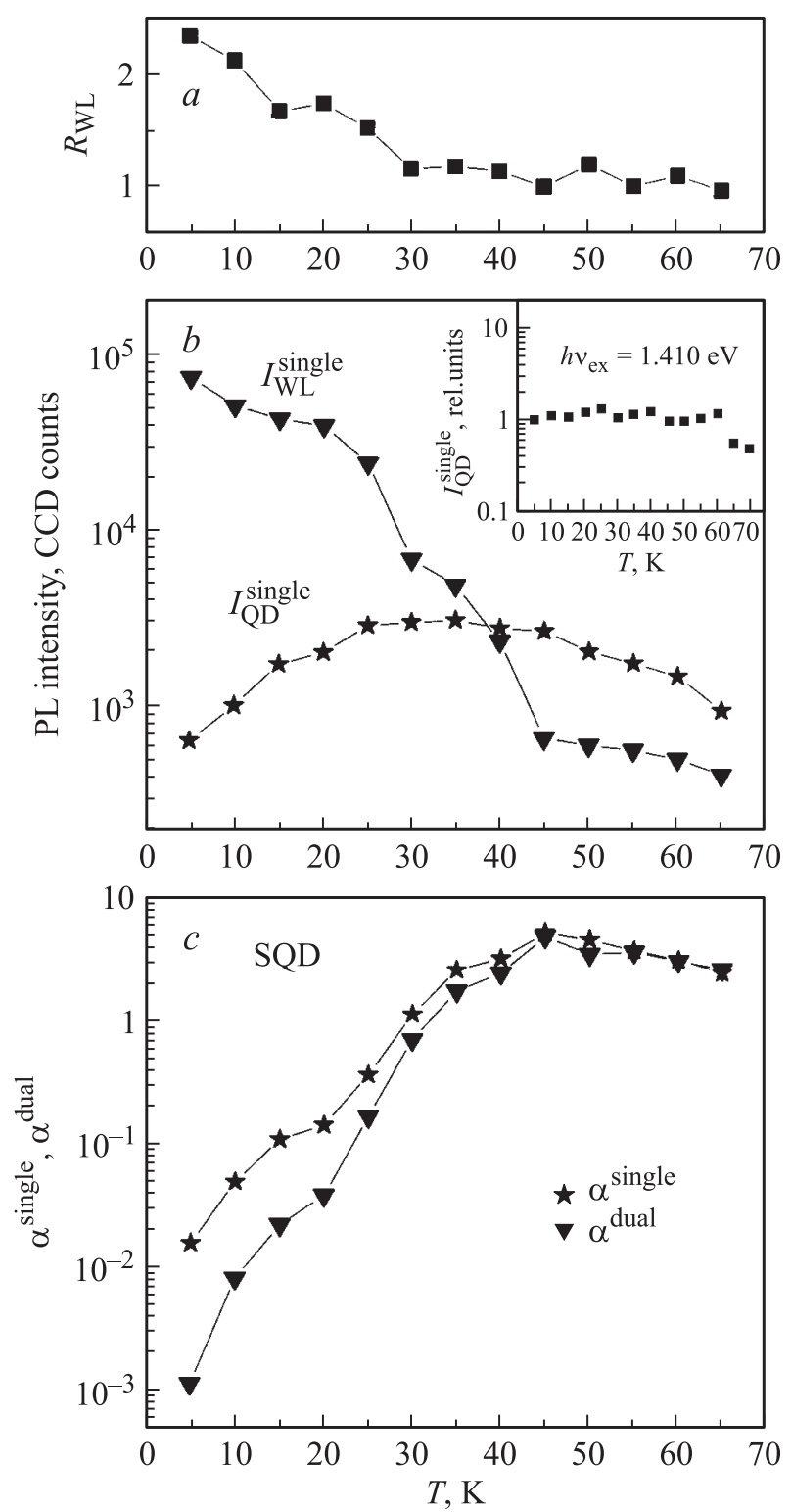

Figure 4. $R_{\mathrm{WL}}(a)$ measured for $\mathrm{SQD}$ at $h v_{\mathrm{IR}}=1.240 \mathrm{eV}$, $h v_{\mathrm{ex}}=1.471 \mathrm{eV}, P_{0}=200 \mathrm{nW}, P_{\mathrm{IR}}=100 \mu \mathrm{W}$ for a number of $T$ 's. $I_{\mathrm{WL}}^{\text {single }}$ and $I_{\mathrm{QD}}^{\text {single }}(b)$ measured for SQD at $h v_{\mathrm{ex}}=1.471 \mathrm{eV}$, $P_{0}=200 \mathrm{nW}$, at different $T$ 's. $\alpha^{\text {single }}$ and $\alpha^{\text {dual }}(c)$ measured for SQD at $h v_{\mathrm{IR}}=1.240 \mathrm{eV}, h v_{\mathrm{ex}}=1.471 \mathrm{eV}, P_{0}=200 \mathrm{nW}$, $P_{\mathrm{IR}}=100 \mu \mathrm{W}$ for a number of $T$ 's. The inset in $(b)$ shows $I_{\mathrm{QD}}^{\text {single }}$ measured for $\mathrm{SQD}$ at $h v_{\mathrm{ex}}=1.410 \mathrm{eV}, P_{0}=17 \mu \mathrm{W}$ at different $T$ 's
$I_{\mathrm{WL}}\left(R_{\mathrm{WL}}>1\right)$. At elevated $T$ 's, the capture to the CNRs becomes more efficient, as explained above. Consequently, the $L_{\mathrm{IR}}$-induced effect of carrier release from the CNRs (arrow $\mathrm{IR}_{1}$ in the inset in Fig. 1), which initiates an essential increase of the $I_{\mathrm{WL}}$ at low $T$ 's, starts to play a minor role at elevated $T$ 's. This explains the gradual decrease of $R_{\mathrm{WL}}$ with increasing $T$ (Fig. 4, $a$ ).

We thus conclude that the observed increase of the $I_{\mathrm{QD}}$ is entirely determined by $T$-induced changes in the transport of carriers. This conclusion is justified by the following experimental observation. At a principal laser excitation energy of $h v_{\text {ex }}=1.410 \mathrm{eV}$ (shown by the vertical solid arrow $h v_{\mathrm{ex}}^{2}$ in the inset in Fig. 1), i. e. at excitation directly into the dot, when no transport of carriers along the plane of the WL is needed prior to capture into the dot, the SQD PL signal does not change in the temperature range $5<T<60 \mathrm{~K}$ (see inset in Fig. 4, $b$ ).

Fig. 4, $c$ shows the experimentally derived values of $\alpha^{\text {dual }}$ and $\alpha^{\text {single }}$ measured for a SQD as a function of temperature. It is seen that both $\alpha^{\text {dual }}$ and $\alpha^{\text {single }}$ progressively increase with increasing $T$, with $\alpha^{\text {dual }} \approx \alpha^{\text {single }}$ at elevated temperatures, which in turn results in the disappearance of the $I_{\mathrm{QD}}$ quenching effect. Consequently, we explain the experimentally observed fact of the progressive increase of the $R_{\mathrm{QD}}$ up to 1 at elevated $T$ 's (Fig. 3,a) in terms of a considerable increase of the QD collection efficiency, rather than by a decrease of $\alpha^{\text {single }}$ down to $\alpha^{\text {dual }}$. In other words, at increased temperatures, the SQD collects carriers so effectively that the role of the internal field which improves the carrier transport at low $T$ 's becomes negligible.

It is interesting to note, that the increase of $T$ results in a qualitatively similar behaviour of $I_{\mathrm{QD}}$ and $I_{\mathrm{WL}}$ measured on high dot density spots. Typical examples of the temperature evolution of $I_{\mathrm{QD}}^{\text {single }}$ measured for the MQDs 1 and MQDs 2 and compared with the SQD are shown in Fig. 3, $b$. An increase of the $I_{\mathrm{QD}}^{\text {single }}$ by approximately 2.7 times was recorded for MQDs 1 which is essentially less than the corresponding value of 5.5 measured for the SQD (Fig. 3, b). In contrast to these observations, only a small increase (up to $50 \%$ ) of the $I_{\mathrm{QD}}^{\text {single }}$ was revealed for MQDs 2. The observed behaviour (Fig. 3,b) is consistent with the idea of a temperature-improved transport of carriers in the plane of the WL. In fact, the different sample positions reveal different values of $\alpha^{\text {single }}$ at $T=5 \mathrm{~K}$, namely $0.01,0.1$ and 2.3 for the SQD, MQDs 1 and MQDs 2, respectively. We note here that in the upper limit of the value of collection efficiency $\left(\alpha^{\text {signle }} \rightarrow \infty\right)$ all the carriers generated are able to capture into the quantum dots even at low $T$. Accordingly, the increase of $T$ is expected to give no further increase of $I_{\mathrm{QD}}$. Consequently, the higher value of $\alpha^{\text {single }}$ at low $T$ is, the less $T$-induced increase of $I_{\mathrm{QD}}$ is predicted, which is nicely confirmed by the data shown in Fig. 3, $b$. These experimental findings are consistent with the temperature-induced behaviour of the $I_{\mathrm{QD}}^{\text {single }}$ revealed in ordinary (macro)-PL measurements: $I_{\mathrm{QD}}^{\text {single }}$ for low dot density revealed an increase by 2 times as $T$ was increased 


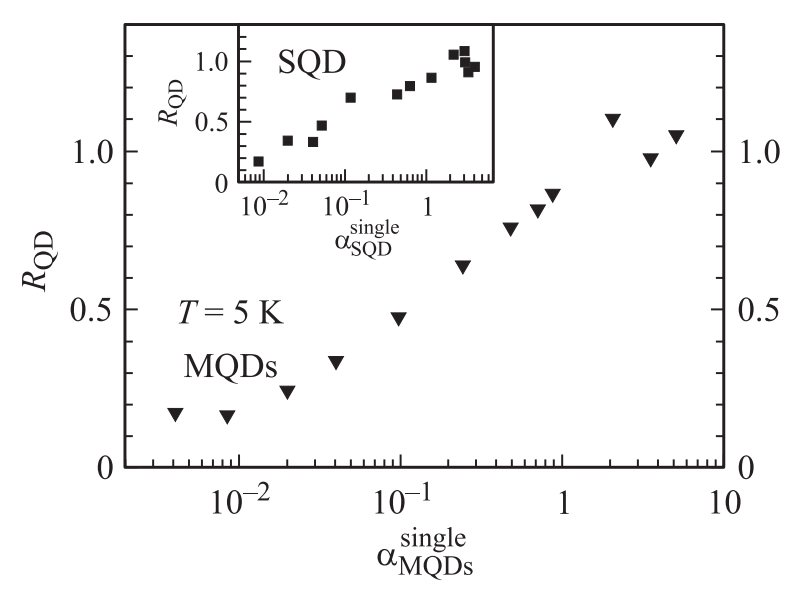

Figure 5. $R_{\mathrm{QD}}$ measured for sample spots with different dot density at $T=5 \mathrm{~K}, h v_{\mathrm{IR}}=1.240 \mathrm{eV}, h v_{\mathrm{ex}}=1.471 \mathrm{eV}, P_{0}=200 \mathrm{nW}$, $P_{\mathrm{IR}}=100 \mu \mathrm{W}$ plotted for a number of $\alpha_{\mathrm{MQDs}}^{\text {single }}$. The inset shows $R_{\mathrm{QD}}$ for SQD plotted for a number of $\alpha_{\mathrm{SQD}}^{\text {single }}$ measured at different $T$ 's (taken from Fig. 4,c).

from 20 to $90 \mathrm{~K}$, while no increase was registered for the case of high dot density structures [14].

The changes in the temperature evolution of the $R_{\mathrm{QD}}$ registered for the case of MQDs 1 and MQDs 2 with respect to the case of SQD (Fig. 3, $a$ ) can also be explained satisfactorily within the model proposed. In fact, with increasing collection efficiency, the role of the internal field becomes less important. This explains the essentially higher values of $R_{\mathrm{QD}}$ 's of 0.5 and 0.9 measured at $T=5 \mathrm{~K}$ for MQDs 1 and MQDs 2, respectively compare to that of 0.1 for the case of SQD (Fig. 3,a). In addition, the higher the collection efficiency is at $T=5 \mathrm{~K}$, the lower temperature is needed to increase its value even more up to the value at which the role of $F$ on the carrier transport diminishes. This explains satisfactorily the $T$ values of 30 and $10 \mathrm{~K}$, at which $R_{\mathrm{QD}}$ becomes equal to 1 for MQDs 1 and MQDs 2 , respectively (Fig. 3, $a$ ).

We finally note that the increase of the $R_{\mathrm{QD}}$ up to 1 , measured for the SQD at different $T$ 's, was interpreted in terms of a temperature-induced increase of the parameter $\alpha^{\text {single }}\left(\alpha^{\text {dual }}\right)$. To verify this interpretation we performed another experiment with the purpose to increase the QDs collection efficiency even at low $T$, namely by selecting sample positions with different dot densities. If the suggested model is correct, a similar effect of $\alpha^{\text {single }}\left(\alpha^{\text {dual }}\right)$ on $R_{\mathrm{QD}}$ should be detected in the latter case as well. Fig. 5 shows the ratio $R_{\mathrm{QD}}$ plotted as a function of $\alpha^{\text {single }}$ for different dot density spots of the structure $\left(\alpha_{\mathrm{MQDS}}^{\text {single }}\right)$ and for the SQD $\left(\alpha_{\mathrm{SQD}}^{\text {single }}\right)$ (inset in Fig. 5). The different values of $\alpha_{\mathrm{SQD}}^{\text {single }}$ were evaluated for different $T$ (from Fig. 4,c), as explained above. The two dependencies behave in a similar way. The progressive increase of $R_{\mathrm{QD}}$ with an increasing $\alpha_{\mathrm{MQDs}}^{\text {single }}$ or $\alpha_{\mathrm{SQD}}^{\text {single }}$ is recorded (Fig. 5 or inset in Fig. 5, respectively), a fact which strongly supports the model proposed.

\section{Conclusion}

An additional IR laser considerably quenches the QD PL signal. This is explained in terms of screening of the internal electric field by the extra holes created in the sample as a result of the IR excitation. The quenching effect progressively vanishes with increasing temperature as well as dot density. These observations are due to a considerably improved QD's collection efficiency at which the effect of electric field on the carrier transport in the plane of the WL becomes less important. The observed effects could be widely used in practice to effectively tune the QD's collection efficiency and manipulating the light emission intensity in QD-based optoelectronic devices.

\section{References}

[1] L. Jacak, P. Hawrylak, A. Wojs. Quantum Dots. SpringerVerlag, Berlin (1998). 176 p.

[2] D. Gammon, D.G. Steel. Physics Today 55, 10, 36 (2002).

[3] L. Harris, D.J. Mawbray, M.S. Skolnick, M. Hopkinson, G. Hill. Appl. Phys. Lett. 73, 7, 969 (1998).

[4] S. Maimon, E. Finkman, G. Bahir, S.E. Schacham, J.M. Garcia, P.M. Petroff. Appl. Phys. Lett. 73, 14, 2003 (1998).

[5] J.J. Finley, M. Skalitz, M. Arzberger, A. Zrenner, G. Bohm, G. Abstreiter. Appl. Phys. Lett. 73, 18, 2618 (1998).

[6] D. Bimberg, M. Grundmann, N.N. Ledentsov. Quantum Dot Heterostructures. Willey, London (1999). 328 p.

[7] K.H. Schmidt, G. Medeiros-Ribeiro, J.M. Garcia, P.M. Petroff. Appl. Phys. Lett. 70, 13, 27 (1997); J.M. Garcia, T. Mankad, P.O. Holtz, P.J. Wellman, P.M. Petroff. Appl. Phys. Lett. 72, 24, 3172 (1998); J.M. Garcia, G. Medeiros-Ribeiro, K. Schmidt, T. Ngo, J.L. Feng, A. Lorke, J. Kotthaus, P.M. Petroff. Appl. Phys. Lett. 71, 14, 2014 (1997).

[8] R. Heitz, M. Veit, N.N. Ledentsov, A. Hoffmann, D. Bimberg, V.M. Ustinov, P.S. Kop'ev, Zh.I. Alferov. Phys. Rev. B 56, 16, 10435 (1997).

[9] A.W.E. Minnaert, A.Yu. Silov, W. van der Vleuten, J.E.M. Haverkort, J.H. Wolter. Phys. Rev. B 63, 7, 075303 (2001); F. Findeis, A. Zrenner, G. Böhm, G. Abstreiter. Phys. Rev. B 61, 16, R10 579 (2000); R. Heitz, I. Mukhametzhanov, O. Stier, A. Madhukar, D. Bimberg. Phys. Rev. Lett. 83, 22, 4654 (1999).

[10] B. Ohnesorge, M. Albrecht, J. Oshinowo, A. Forchel, Y. Arakawa. Phys. Rev. B 54, 16, 11532 (1996); U. Bockelman, T. Egeler. Phys. Rev. B 46, 23, 15574 (1992); A. Rack, R. Wetzler, A. Wacker, E. Schöll. Phys. Rev. B 66, 16, 165429 (2002); S. Raymond, K. Hinzer, S. Fafard, J.L. Merz. Phys. Rev. B 61, 24, 16331 (2000).

[11] P.P. Paskov, P.O. Holtz, B. Monemar, J.M. Garcia, W.V. Schoenfeld, P.M. Petroff. Appl. Phys. Lett. 77, 6, 812 (2000).

[12] Y. Toda, O. Moriwaki, M. Nishioka, Y. Arakawa. Phys. Rev. Lett. 82, 20, 4114 (1999).

[13] A.F.G. Monte, J.J. Finley, A.D. Ashmore, A.M. Fox, D.J. Mowbray, M.S. Skolnick, M. Hopkinson. J. Appl. Phys. 93, 6, 3524 (2003).

[14] C. Lobo, R. Leon, S. Marcinkevičius, W. Yang, P. Sercel, X.Z. Liao, J. Zou, D.J.H. Cockayne. Phys. Rev. B 60, 24, 16647 (1999). 
[15] M.M. Sobolev, A.R. Kovsh, V.M. Ustinov, A.Yu. Egorov, A.E. Zhukov, M.V. Maksimov, N.N. Ledentsov. Semicond. 31, 10, 1074 (1997).

[16] S. Marcinkevičius, J. Siegert, R. Leon, B. Čechavičius, B. Magness, W. Taylor, C. Lobo. Phys. Rev. B 66, 23, 235314 (2002).

[17] S. Ménard, J. Beerens, D. Morris, V. Aimez, J. Beauvais, S. Fafard, J. Vac. Sci. Technol. B 20, 4, 1501 (2002).

[18] S. Marcinkevičius, A. Gaarder, R. Leon. Phys. Rev. B 64, 11, 115307 (2001).

[19] S. Marcinkevičius, R. Leon. Appl. Phys. Lett. 76, 17, 2406 (2000).

[20] P.W. Fry, J.J. Finley, L.R. Wilson, A. Lemaitre, D.J. Mowbray, M.S. Skolnick, M. Hopkinson, G. Hill, J.C. Clark. Appl. Phys. Lett. 77, 26, 4344 (2000).

[21] E.S. Moskalenko, V. Donchev, K.F. Karlsson, P.O. Holtz, B. Monemar, W.V. Schoenfeld, J.M. Garcia, P.M. Petroff. Phys. Rev. B 68, 15, 155317 (2003).

[22] P. Silverberg, P. Omling, L. Samuelson. Appl. Phys. Lett. 52, 20, 1689 (1988).

[23] D.A. Mazurenko, A.V. Scherbakov, A.V. Akimov, A.J. Kent, M. Henini. Semicond. Sci. Technol. 14, 12, 1132 (1999).

[24] M. Sugisaki, H.W. Ren, K. Nishi, Y. Masumoto. Phys. Rev. Lett. 86, 21, 4883 (2001).

[25] E.S. Moskalenko, K.F. Karlsson, P.O. Holtz, B. Monemar, W.V. Schoenfeld, J.M. Garcia, P.M. Petroff. Phys. Rev. B 64, 8,085302 (2001).

[26] A.V. Akimov, V.V. Krivolapchuk, N.K. Poletaev, V.G. Shofman. Semicond. 27, 2, 171 (1993).

[27] K.F. Karlsson, E.S. Moskalenko, P.O. Holtz, B. Monemar, W.V. Schoenfeld, J.M. Garcia, P.M. Petroff. Appl. Phys. Lett. 78, 19, 2952 (2001).

[28] H.D. Robinson, B.B. Goldberg. Phys. Rev. B 61, 8, R5086 (2000).

[29] J. Seufert, R. Weigand, G. Bacher, T. Kummell, A. Forchel, K. Leonardi, D. Hommel. Appl. Phys. Lett. 76, 14, 1872 (2000).

[30] V.N. Abakumov, V.I. Perel, I.N. Yassievich. Nonradiative Recombination in Semiconductors. North-Holland, Amsterdam (1991). 320 p. 